\title{
tic\&société
}

Vol. 10, N 2-3 | 2ème semestre 2016 - 1er semestre 2017

Spécial varia

\section{Une dialectique de la pudeur : les pratiques de mise en visibilité de soi sur Facebook}

\section{Laurent MELL}

\section{(2) OpenEdition \\ 1 Journals}

\section{Édition électronique}

URL : http://journals.openedition.org/ticetsociete/2088

DOI : 10.4000/ticetsociete. 2088

\section{Éditeur}

Association ARTIC

Édition imprimée

Pagination : 45-70

\section{Référence électronique}

Laurent MELL, "Une dialectique de la pudeur : les pratiques de mise en visibilité de soi sur Facebook », tic\&société [En ligne], Vol. 10, № 2-3 | 2ème semestre 2016 - 1er semestre 2017, mis en ligne le 30 avril 2017, consulté le 19 avril 2019. URL : http://journals.openedition.org/ticetsociete/2088 ; DOI : 10.4000/ticetsociete.2088 
tic\&société - 10(2-3), 2016-2017

\section{Une dialectique de la pudeur : les pratiques de mise en visibilité de soi sur Facebook}

\section{Laurent MELL}

Laurent Mell est docteur en Sciences de l'information et de la communication au CEMTI (Centre d'études sur les médias, les technologies et l'internationalisation) à l'Université Paris 8. II est actuellement ingénieur de recherche au LABERS (LABoratoire d'études et de recherches en sociologie) à l'Université de Bretagne Occidentale (UBO) à Brest. Ses recherches s'articulent autour de trois axes. Le premier porte sur les usages des technologies de l'information et de la communication, sur les différentes formes de mise en visibilité numérique de soi ainsi que sur la place de l'esthétique et de la pudeur dans ces pratiques. Le deuxième traite de la relation entre numérique, pédagogie et innovation. Un troisième axe se concentre sur le rapport des jeunes aux médias et à l'actualité médiatique. laurent.mell@hotmail.fr 


\section{Une dialectique de la pudeur: les pratiques de mise en visibilité de soi sur Facebook}

Résumé : L'amplification des usages des technologies de l'information et de la communication (TIC), et plus particulièrement des réseaux socionumériques, ont induit des évolutions significatives dans le rapport des individus aux normes relatives à la pudeur. Dans cet article, nous proposons de discuter des pratiques de mise en visibilité de soi sur le réseau socionumérique Facebook. Tout d'abord, nous montrons que l'augmentation de la considération pour la vie privée amène à une sélection des informations divulguées et à une fragmentation de ce que les usagers donnent à voir à autrui. Ensuite, nous soulignons le caractère dynamique de ces pratiques au travers de diverses temporalités de mise en visibilité de soi. Enfin, nous concluons sur l'évolution du cadre normatif de la pudeur en soulignant que les usages de Facebook intègrent dorénavant davantage de jeu avec les règles régulant la mise en visibilité de soi, du corps et des sentiments.

Mots-clés: visibilité, réseaux socionumériques, pudeur, usages, intimité.

Abstract: The increasing use of information and communication technologies (ICTs) particularly social networking sites, has induced significant changes in the relationship of individuals to standards of decency. In this article, we discuss self-exposure practices on the social networking site Facebook. First of all, we observe that increasing concerns about privacy leads to selective disclosure of information and a fragmentation of what users show to others. Secondly, we emphasize the dynamic nature of these practices through various temporalities of self-exposure. Finally, we conclude on the evolution of the normative framework of modesty by pointing out that Facebook users now play more with the rules governing the visibility of self, body and feelings.

Keywords: visibilty, social networking site, decency, uses, intimacy.

Resumen: El uso creciente de las tecnologías de la información y de la comunicación (TIC), y en particular de las redes sociales, ha significado cambios significativos en la relación que los individuos mantienen con las normas relativas 
Une dialectique de la pudeur : les pratiques de mise en visibilité de soi sur Facebook

al pudor. El presente artículo propone analizar las prácticas de auto-exposición en Facebook. En primer lugar, se muestra que los cambios en la consideración de lo que es vida privada conduce a una selección de las informaciones divulgadas y a una fragmentación de aquello que los usuarios ofrecen para que sea visto por otras personas. Posteriormente, se hace hincapié en el carácter dinámico de estas prácticas a través de diferentes temporalidades de la visibilidad del individuo. Por último, se concluye sobre la evolución del marco normativo del pudor subrayando que los usos de Facebook contribuyen a crear las reglas que regulan la auto-exposición del cuerpo y de los sentimientos.

Palabras clave : visibilidad, redes sociales, pudor, usos, intimidad. 


\section{Introduction}

Durant une longue période de l'histoire des sociétés occidentales, la publicité de l'intimité était assimilée à un acte inapproprié aux mœurs en vigueur. Le relâchement progressif des individus face aux règles de la pudeur ainsi que les usages toujours croissants des récents dispositifs sociotechniques conduisent à considérer de nouveau le rapport au privé et à l'intime. Dans cet article, nous présentons une partie des résultats de notre thèse portant sur les pratiques de mise en visibilité de soi sur Facebook. Dans le cadre de cette recherche, nous avons cherché à comprendre, suivant un raisonnement dialectique, les raisons expliquant la composition de pratiques de régulation de la visibilité numérique face à un réseau socionumérique invitant à une exposition de soi accrue. Notre souci est de mettre en évidence des phénomènes agissant sur ces pratiques de mise en visibilité de soi, de nous saisir de ce qui se présente a priori comme des contradictions et de les dépasser afin de les expliquer.

Dans le contexte de cet article, nous entendons la dialectique comme un mode de raisonnement procédant par la confrontation d'idées a priori contradictoires en vue de les surmonter et de souligner leur corrélation. Nous souhaitons penser conjointement des a priori contradictoires, notamment concernant les dichotomies public/privé et pudeur/indécence, dans le but d'affiner la compréhension des pratiques de mise en visibilité de soi sur Facebook.

Les méthodes d'enquête employées dans le cadre de cette recherche sont de deux ordres : une enquête qualitative et une enquête quantitative ont été menées en parallèle. Concernant l'enquête quantitative, une invitation à répondre à un questionnaire en ligne a été diffusée, dans le courant de l'année 2011, auprès d'usagers suivant l'actualité de la page Facebook de la marque Hénaff ${ }^{1}$. Cette sollicitation a permis de recueillir 444 réponses au questionnaire dont les données ont ensuite été traitées à l'aide du logiciel Sphinx. Concernant l'enquête qualitative, des entretiens semi-directifs ont été réalisés auprès d'acteurs et d'usagers de six structures associatives de quartiers de la ville de Brest (centre social, maison des jeunes et de la culture, maison pour tous, maison de quartier). Une série de 27 entretiens ( 25 en présence et

\footnotetext{
${ }^{1}<$ https://www.facebook.com/patehenaff>, dernière consultation le 16 mars 2017.
} 
Une dialectique de la pudeur : les pratiques de mise en visibilité de soi sur Facebook

deux à distance en visioconférence) a été effectuée de novembre 2013 à avril 2014.

Dans un premier temps, nous rappellerons que la massification d'usages des réseaux socionumériques a entraîné, ces dernières années, la focalisation de l'intérêt de chercheurs sur le sujet, conduisant, par la même, à l'émergence de questions autour de l'exposition de soi et de la mise en visibilité sur Internet. Par ailleurs, la prise de conscience par les usagers de la visibilité informationnelle sur les réseaux socionumériques les a amenés à revoir les rapports qu'ils entretiennent avec autrui et à élaborer des stratégies en vue de gérer leur représentation numérique. Les normes propres aux mondes numériques, à savoir qu'elles engagent à une révision du rapport "traditionnel " au temps et à l'espace, bousculent l'ancrage de l'individu dans l'espace public. L'accroissement du souci pour la vie privée combiné à un dévoilement plus ou moins négocié d'une partie des informations personnelles sont l'expression d'un déplacement de l'espace d'intimité et des temporalités de mise en visibilité de soi sur les réseaux socionumériques. Suivant une approche diachronique, ces pratiques s'inscrivent dans une société a priori perçue comme plus "permissive », où la privatisation croissante des fonctions corporelles, assimilée à une relégation des désirs dans les tréfonds de l'intimité, conduit tout un chacun à réévaluer régulièrement son rapport à l'intime, au privé, mais aussi au public.

Dans un second temps, nous présenterons une réflexion s'appuyant sur les résultats de notre recherche en nous attardant sur la question de la fragmentation de la visibilité informationnelle sur Facebook, entendue dans ce contexte comme une décomposition des informations concernant les caractéristiques identitaires de l'usager sur le réseau socionumérique. Cette répartition des accès aux informations personnelles renseignant le profil de l'usager suivant trois espaces de visibilité entre dans une dynamique d'intensification du rapport à soi et apparaît comme une pratique de modération de la visibilité dans un souci de soi. Le caractère dynamique de ces pratiques induit une réorganisation, plus ou moins régulière, du visible par l'élaboration de stratégies de mise en visibilité de soi, suivant l'ancienneté d'usage. L'expérience accumulée par l'usager dans le temps devient un facteur de variation de la taille de ces espaces de visibilité informationnels. À côté de cela, il semble que les facteurs d'invitation à l'exposition de soi correspondent, en grande partie, aux mécanismes de préservation contre les potentielles atteintes à 
la vie privée. Ces facteurs agissent dans un contexte de révision du rapport au privé, au public, mais aussi à la pudeur.

\section{Le phénomène de visibilité numérique}

Ces dernières années, l'intérêt des chercheurs en sciences sociales pour les réseaux socionumériques a conduit un certain nombre d'entre eux à interroger les pratiques d'exposition de soi et de mise en visibilité sur Internet (Aguiton et al., 2009 ; Denouël, 2011). Prenant appui sur les travaux de Laurence Allard et de Frédéric Vandenberghe (2003), Alexandre Coutant et Thomas Stenger (2010) ont montré que les réseaux socionumériques participent au développement d'une culture " expressiviste " et que s'exercent, en leur sein, différentes formes de contrôle social et d'exploitation de la participation. D'ailleurs, maintes recherches s'appuient sur celles qui ont été, depuis un certain temps, réalisées par Danah Boyd (2008b) et Nicole Ellison (Boyd et Ellison, 2007). Nombreux sont les usagers à réaliser le degré de visibilité de leurs informations sur les réseaux socionumériques et à infléchir leur rapport à autrui.

La variété des publics auxquels l'usager peut s'adresser l'amène à disposer ses informations personnelles dans des "zones grises ", des espaces oscillants entre l'« absolument secret » et le " complètement public ». Les modes d'interaction, spécifiques aux technologies numériques de communication, ont induit une révision de la relation de l'usager au temps et à l'espace. Cette invitation à l'exposition numérique tend à considérer l'identité en ligne comme un « vaste bazar où tout semble faire signe » (Cardon, 2008, p. 95).

En conséquence, nombre d'études se sont concentrées sur l'influence que peuvent exercer les TIC sur les "jeunes» utilisateurs, sur la manière dont ces derniers s'en saisissent et sur le rapport que peuvent avoir les "adultes" sur ce phénomène (Ito et al., 2008). Cette jeune population, fortement confrontée à ces dispositifs de communication, subit de rapides et successifs bouleversements de sa visibilité dans l'espace public numérique (Boyd, 2007, 2014). L'adoption des réseaux socionumériques par les adolescents a fait naître de nouvelles interrogations concernant l'expression de leurs caractéristiques identitaires, la visibilité de celles-ci et les conséquences sur les normes interactionnelles en vigueur. 
Une dialectique de la pudeur : les pratiques de mise en visibilité de soi sur Facebook

Face à la complexité architecturale et d'usage des réseaux socionumériques, les usagers ont été contraints d'élaborer des stratégies en vue de gérer leur représentation numérique (Boyd, 2008a). Constamment sollicités, ces utilisateurs développent des pratiques d'actualisation de soi, négociant entre les opportunités offertes et les risques encourus (Boyd et Hargittai, 2013 Livingstone, 2008). Entre un souci individuel de protection de l'intimité et une économie d'exploitation des données personnelles, les usagers façonnent des techniques afin de parer aux inquiétudes liées à la gestion de l'identité numérique (Lenhart et Madden, 2007).

Ce que les TIC ont introduit dans ce mode de communication médiatisée, c'est ce tiers en capacité de s'immiscer dans l'interaction (Cardon et Delaunay-Teterel, 2006). Les échanges communicationnels effectués sur Internet font intervenir, audelà de la personne qui parle et de celle qui écoute, une troisième personne, un tiers plus ou moins attentif, un public témoin. L'extension de la visibilité des individus en interaction et des informations échangées fait qu'il est désormais possible à un public tiers de participer, tout du moins de se positionner en tant que spectateur. Ce tiers, dont l'anonymat varie selon le dispositif sociotechnique mobilisé, est un levier d'inflexion primordial quant à l'intervention des usagers sur cette " hiérarchie du voir » (Voirol, 2005).

\section{Des temporalités et des espaces d'exposition}

La pratique de mise en visibilité de soi implique nécessairement une évolution au cours du temps. Celui-ci se pose alors en élément fondamental dans la construction et la modulation de ces pratiques. Dans le cadre de cette recherche, nous pensons qu'il est possible d'élaborer une chronologie des temporalités de mise en visibilité numérique de soi. Ce faisant, nous allons nous attacher à développer notre réflexion à partir d'un temps court et, plus particulièrement, à partir de temporalités de gestion de la visibilité individuelle sur les réseaux socionumériques. Ces temporalités renvoient ici à des constructions subjectives organisées selon des pratiques. De fait, nous partons du principe que la temporalité est une construction sociale non indépendante du raisonnement individuel et fondée sur la pratique.

De manière synthétique, nous nous entendons sur l'idée que le temps est le «lieu d'intelligibilité des phénomènes » (Dubar 
et Rolle, 2008). L'École des Annales s'est d'ailleurs attachée à différencier plusieurs niveaux d'analyse du temps: le temps long comme celui des évolutions géographiques ou économiques; le temps moyen s'étalant au niveau des institutions et des biographies; et le temps court traitant de l'événement et du vécu (Dubar et Thoemmes, 2013). Le monde repose sur des processus physiques - qu'ils soient dépendants ou non de l'homme - et sur des interprétations individuelles de la succession des évènements (Elias, 1996). Le temps ne peut uniquement se réduire à une simple construction humaine ou à une dynamique extérieure échappant à l'individu.

Nous faisons le choix de penser les temporalités comme "des "domaines" de structuration du temps " et non comme des moments du temps passé, présent ou futur (Dubar et Rolle, 2008, parag. 11). En cela, elles nous permettent de focaliser notre réflexion sur l'évolution d'un fait social spécifique à travers l'écoulement du temps. Nous comprenons la notion de temporalité davantage dans une approche contextuelle, c'est-àdire que nous insistons sur la place de l'individu et de son environnement dans le temps (Samuel, 2008). Le temps et l'espace coexistent puisque tout changement dans l'espace implique un changement dans le temps, et l'inverse vaut tout aussi bien (Heinich, 1997).

II peut paraître inconfortable d'employer le terme d'espace pour un environnement virtuel comme Internet, dans le sens où il est souvent perçu comme a-spatial (Beaude, 2012). Pour autant, il correspond indéniablement à un espace réel d'interactions sociales qui «participe pleinement de la spatialité [dimension spatiale de l'action] contemporaine " (Beaude, 2012, p. 18). La multitude des échanges entretenus dans ce « milieu » fait qu'ils occupent bel et bien un espace. La création, le prolongement et le développement de relations sociales dans cet espace soulignent son caractère pérenne et sa réalité. Par ailleurs, la multiplication des espaces de communication et la diversification des applications ont complexifié la gestion des espaces personnels.

\section{Frontières du privé et négociations de la pudeur}

Le développement des technologies de communication influence nos manières d'échanger et agence, d'une façon nouvelle, les espaces de conversation. Ce phénomène introduit une révision du rapport au privé et à l'intime dans le contexte 
Une dialectique de la pudeur : les pratiques de mise en visibilité de soi sur Facebook

d'usage de ces nouvelles technologies. L'intérêt progressif des individus pour leur intimité lié à des pratiques plus ou moins maîtrisées de mise en visibilité de soi sont les symptômes de l'évolution des espaces privé et public sur les réseaux socionumériques et, plus largement, sur Internet. La société contemporaine "vide l'espace public sous l'effet d'un impératif de transparence généralisée » (Akoun, 1998, p. 119). Les individus se retournent vers les sphères privées, notamment la famille, avec une forte volonté de protection de l'intimité. Richard Sennett (1979) tend même à déclarer que " plus cette tyrannie de l'intimité s'impose, plus les gens cherchent à se libérer des coutumes, des manières sociales, des codes, etc., pour s'ouvrir de façon inconditionnée les uns aux autres » (p. 274). Néanmoins, nous doutons du caractère tyrannique de l'intimité et pensons qu'il s'opère un nivellement entre les différents espaces de visibilité. Les réseaux socionumériques amènent les individus à sectoriser leur espace d'interactions et, par extension, à tenter de délimiter leur espace de visibilité.

Depuis quelques siècles, il s'est opéré une différenciation de plus en plus prononcée entre la vie privée et la vie publique, correspondant à ce que Roger Chartier (1999) définit comme la civilité, c'est-à-dire le "comportement de l'individu en société » (p. 162). Ce dernier doit reléguer l'ensemble de ses émotions dans l'espace intime et adopter un comportement raisonné dans l'espace public. L'accroissement du fossé qui sépare la vie privée de la vie publique, concomitant de la privatisation de l'espace public, participe à l'émergence et à la structuration de la civilisation (Perrot, 1999). Dans l'antiquité, les Romains éprouvaient des difficultés à faire la distinction entre "fonctions publiques et dignité privée, finances publiques et bourse personnelle » (Ariès et Duby, 1999, p. 95). À l'inverse, durant la période du haut Moyen Âge, la vie privée est privilégiée, dans le sens où « il faut cacher l'enfance, ces années si fragiles, vivre en privé, pour éviter le malheur " (Ariès et Duby, 1999, p. 421). En érigeant de telles protections, il s'est opéré un repliement sur la famille comme une volonté d'occulter le regard d'autrui, sinon de l'amoindrir. Bien qu'il y ait eu une augmentation des considérations pour la vie privée durant ces quelques siècles, à l'instar de notre période contemporaine, ces choix n'ont pas nécessairement été appliqués pour les mêmes raisons. À certaines époques, il s'agissait de préserver l'innocence des vicissitudes du monde extérieur, mais, aujourd'hui, ce phénomène est davantage une réponse à l'accroissement de l'individualisme. L'augmentation de la considération de l'individu pour sa condition l'a amené à revoir la place quotidienne du soi dans la société. 
Bien que la conception de la vie privée, dans le sens où nous l'entrevoyons aujourd'hui, soit assez récente, il n'en demeure pas moins qu'il existe une myriade de définitions, tant philosophiques que juridiques. Cela peut s'expliquer par le flou qui règne autour des concepts et des notions vers lesquels ce terme renvoie: légitimité, non-ingérence, défense du secret, intimité, respect, secret, confidentialité, etc. (Polin, 2000; Tabatoni, 2002). Cette liste n'est en rien exhaustive, mais elle est l'expression des difficultés rencontrées dans la définition de la vie privée (Altman, 1977; Fried, 1970 ; Froomkin, 2000 ; Kang, 1998 ; Stone et al., 1983 ; Warren et Brandeis, 1890 ; Westin, 1967). Celle qui, à l'heure actuelle, nous semble la plus pertinente correspond à une "décision idiosyncrasique de tracer la frontière entre existence personnelle et environnement social ॥ (Vincent, 1999, p. 551). Cette frontière amène à penser la vie privée davantage en termes contextuels qu'informationnels. Pierre Tabatoni (2000), en reprenant les propos de François Rigaux, apporte une précision dans la compréhension de la vie privée en expliquant "qu'il est impossible, et au surplus inutile, de définir la vie privée, et le mur (de la vie privée) n'est qu'une limite stratégique qui se déplace au gré des circonstances » (p. 4).

Historiquement, du fait de la sensibilité qui entoure l'exposition du corps ou des sentiments, il existe relativement peu de textes qui s'étendent sur le sujet de la pudeur. Norbert Elias (1973), mobilisant les écrits d'Érasme, rappela que le $\mathrm{XV}^{\mathrm{e}}$ siècle fut une période charnière quant à l'évolution du seuil de la pudeur et qu'antérieurement "les actes eux-mêmes, les propos auxquels ils donnent lieu, les associations d'idées qui les accompagnent n'ont pas encore ce caractère "intime" et "privé" qui en fera plus tard des objets de pudeur et de sensations de malaise » (p. 192). Nous entendons, d'ailleurs, la notion de pudeur comme le
"sentiment qui retient d'accomplir ou de regarder toute action (pudeur corporelle) ou sa représentation (pudeur artistique) condamnée par un code moral personnel (pudibonderie) ou caractéristique d'une époque et d'un lieu donnés (pudeur), par respect pour soi-même (pudeur) ou pour les autres (décence) » (Bologne, 1997, p. 20).

Nos gestes, a priori considérés comme étant " naturels », sont élaborés suivant des normes collectives. L'installation de l'ensemble de ces seuils de pudeur et de honte a été un tournant dans l'histoire du rapport au corps et aux sentiments parce que « ces contrôles corporels lentement élaborés, vite oubliés pourtant au point d'en apparaître naturels, contribuent 
Une dialectique de la pudeur : les pratiques de mise en visibilité de soi sur Facebook

par leur "incorporation" même à "modeler en retour la sensibilité" " (Corbin, Courtine et Vigarello, 2005, p. 11). Norbert Elias (1991) voit en cela un "très haut degré de réserve, de contrôle des réactions affectives, d'inhibition ou de transformation de l'instinct » (p. 65). En ce sens, l'exposition du corps doit susciter une sensation de gêne ou d'angoisse, tout du moins le sentiment de plaisir, issu de l'exposition de la nudité, doit être relégué en l'espace intime. Pour autant, il s'opère aujourd'hui un certain relâchement par rapport aux règles de la pudeur.

Une société, a priori perçue comme plus "permissive », s'inscrit davantage dans une évolution du rapport aux normes intériorisées, permettant une liberté plus importante (Heinich, 1997). Norbert Elias (1973) a souligné que la privatisation croissante des fonctions corporelles, assimilée à une relégation des désirs dans les tréfonds de l'intimité, a amené à une réévaluation du rapport à l'intime, au privé et au public. Or, là aussi, ce qu'il révélait a évolué et tend à renvoyer à une déprivatisation des fonctions corporelles, tout en maintenant l'individu au cœur d'un paradoxe, celui du confinement des instincts et des pulsions dans une société valorisant les pratiques de séduction et la sexualisation des attributs corporels.

\section{Des pratiques de modération de la visibilité dans un souci de soi}

L'ensemble des outils numériques à disposition de l'usager quant à la modulation identitaire ne sont-ils pas un symptôme de ce que Claude Dubar (2010) appelle la crise des identités ? Dans la constitution de l'identité numérique, il semble que

« les crises [soient] au cœur de cette construction, toujours fragile et inachevée, d'un sujet plongé dans une forme sociale à dominante "sociétaire" [...] impliquant l'établissement de liens sociaux volontaires et incertains par contraste avec les individus socialisés de manière d'abord communautaire » (Dubar, 2010, p. 163).

Ce sont autant de manières de se présenter, mais aussi de ne pas se présenter à autrui. Selon la quantité d'informations dévoilées et le degré de véracité des propos véhiculés, il est plus ou moins aisé de distinguer la personne de ses alter ego. Ce phénomène n'a rien de nouveau, mais, avec le 
développement des TIC, il est dorénavant permis à tout un chacun de s'exprimer sous une multitude d'identités sur une myriade de supports. La pratique de ces dispositifs sociotechniques, oscillant entre l'affirmation de son individualité et la recherche de sociabilité, réclame dorénavant une présentation attentive de soi.

L'analyse quantitative de nos données a permis de dresser une échelle de mesure du degré de divulgation des informations structurant le profil de l'usager de Facebook et, ainsi, de discuter des potentielles modulations de la visibilité informationnelle. La majorité des personnes interrogées considère les préférences sexuelles (64\%), le courriel $(64 \%)$, la religion $(70 \%)$, l'orientation politique $(73 \%)$ et le numéro de téléphone $(93 \%)$ comme étant des informations personnelles, entendues comme restreintes à leur seule personne. Ce ne sont pas les seules informations analysées, mais les plus significatives. Des résultats complémentaires montrent qu'il apparaît trois espaces de visibilité : l'espace intime, l'espace commun et l'espace public. Le premier, l'espace intime, correspond à un espace exclusif d'accès à certaines informations personnelles (numéro de téléphone, religion, opinion politique, relation recherchée, préférence sexuelle). Le second, l'espace commun, renvoie à un espace restreint d'accès (situation amoureuse, domicile, lieu de naissance, courriel, études, emploi, anniversaire, centres d'intérêt), non exclusif sans pour autant être public, qui concerne principalement le réseau de relations de l'usager. Le troisième, l'espace public, représente un espace public (sexe) non restreint aux autres usagers, entendu comme ouvert à tous les internautes. Les usagers sont en mesure de développer des espaces de visibilité de leurs informations personnelles et d'ainsi créer des frontières afin d'administrer les accès.

Ces pratiques de modération de la visibilité contredisent les lieux communs concernant l'exposition de soi totalement dérégulée. L'augmentation de la considération pour la vie privée amène à une sélection des informations divulguées à autrui. Cette distance maintenue vis-à-vis de cette invitation à la mise en visibilité de soi conduit les individus à réévaluer, plus ou moins régulièrement, la valeur et la place occupée par leurs informations dans les espaces de visibilité. Par ailleurs, ces trois espaces de visibilité renvoient à ce contrôle social du territoire du moi, qu'Erving Goffman (1973) explique en ces mots: "chaque fois que des individus se trouvent en présence [d'autrui], leurs territoires du moi tendent sur la scène un vaste filet de clôtures que chacun d'eux est particulièrement bien équipé pour franchir »(p.110). Les individus établissent des 
Une dialectique de la pudeur : les pratiques de mise en visibilité de soi sur Facebook

barrières de protection du moi en sachant qu'elles ne sont absolument pas infranchissables. Ces trois espaces de visibilité comportent des "clôtures » plus ou moins perméables avec des mobilités entre leurs contenus suivant le contexte de communication.

Cette répartition des accès aux informations personnelles entre dans une dynamique d'intensification du rapport à soi, que Michel Foucault (1984) entend comme « des formes dans lesquelles on est appelé à se prendre soi-même pour objet de connaissance et domaine d'action, afin de se transformer, de se corriger, de se purifier, de faire son salut » (p. 56). Mais estce que tous les usagers de Facebook éprouvent un réel « souci de soi "? Les exigences d'austérité sexuelle fortement exprimées du temps de l'Empire romain se caractérisent par le développement de ce que Foucault appelle la "culture de soi ॥, amenant à une valorisation du rapport de soi à soi. Ce renforcement de l'austérité sexuelle, dans l'Antiquité, doit moins être perçue comme un système de restrictions et de définition des prohibitions que comme un renforcement de l'intérêt pour soi, tant sur les plans du corps que de l'esprit. Cette époque et la nôtre sont distantes temporellement. Toutefois, nous pouvons transposer ce processus aux usages des réseaux socionumériques et envisager la culture de soi comme permanente à nos sociétés contemporaines. Par ailleurs, la culture de soi n'est pas un "exercice de solitude », mais une "véritable pratique sociale », sous-entendant qu'autrui est nécessaire à l'exploitation de la culture de soi (Foucault, 1984, p. 67). Dès lors, la diversité des pratiques de mise en visibilité de soi sur Facebook ne s'oppose pas à la valorisation du rapport à soi. La répartition des informations personnelles, suivant ces trois espaces de visibilité, apparaît donc comme une pratique de modération de la visibilité de soi dans un souci de soi.

La culture de soi est un principe qui existait déjà à l'époque des contemporains de Xénophon aux alentours de 400 avant J.-C. (Foucault, 1984). Ayant évoluée depuis, la culture de soi renvoie dorénavant à des formes d'individualisme ou d'égoïsme, à un amour de soi inconsidéré et quelque peu suspect. Cette culture de soi, dont Foucault met en avant les mécaniques dans l'Empire romain, a subi de profonds changements. Là où, jadis, elle se posait en condition nécessaire à une meilleure compréhension et maîtrise de soi, elle le semble aujourd'hui d'autant moins. II serait pertinent d'interroger, dans un travail de recherche à venir, les moteurs ainsi que les finalités de cette culture de soi dans ces pratiques de mise en visibilité numérique. Nous pourrions alors nous 
demander si les pratiques de mise en visibilité de soi sur les réseaux socionumériques correspondent véritablement à une culture de soi. L'exposition de caractéristiques identitaires, sous des formes diverses (texte, photo, vidéo, etc.) sur Facebook, répond-elle à un besoin de connaissance et de perfectionnement de soi, ou n'est-ce qu'une culture de l'ego avec l'attribution d'une valeur absolue à l'individu par rapport à ses groupes d'appartenance et aux institutions? Ou encore, suivant un raisonnement dialectique, n'est-ce pas dans la négociation permanente de l'équilibre entre ces deux attentes que les pratiques de mise en visibilité numérique de soi se situent?

\section{Des temporalités de mise en visibilité de soi}

Une majorité des personnes interrogées se sont inscrites à Facebook et se connectent régulièrement par imitation d'autrui. II transparaît un véritable besoin de respecter les normes en vigueur, de ne pas les transgresser, de ne pas entrer dans une " anormalité » sociale. La massification de l'usage de Facebook, tant en nombre d'usagers que sur le temps passé, positionne ce dispositif sociotechnique en nouvel espace de sociabilité. Cet espace conversationnel supplémentaire renvoie à un besoin de connexions amicales permanent, ce que Boyd (2014) appelle la « contrainte de l'amitié ».

Les temporalités se posent alors en jalon vers la compréhension de la visibilité des individus sur Facebook. Les pratiques d'actualisation de soi sur les réseaux socionumériques intègrent un processus temporel d'usages comprenant des "phases » de création et de recréation identitaire. Et, à l'intérieur de ce processus, le temps joue un rôle majeur dans la construction et la modulation de ces pratiques. L'élaboration de l'identité en ligne correspond à un continuum entrecoupé de divers épisodes de vie (Denouël, 2011). Plus spécifiquement, ces propos visent à comprendre l'identité numérique comme une écriture de soi et une mise en intrigue de ce qui nous différencie ou nous rapproche d'autrui. Elle s'apparente à un continuum individuellement et socialement construit.

Tout en étant nombreux à être préoccupés par la persistance informationnelle sur les réseaux socionumériques et, plus spécifiquement, sur Facebook, il n'en demeure pas moins qu'une majorité des personnes interrogées ont un usage de Facebook sur leur lieu de travail ou d'études. Dans le cadre 
Une dialectique de la pudeur : les pratiques de mise en visibilité de soi sur Facebook

professionnel, ces usages de Facebook s'opposent à ce qui est socialement attendu, à savoir une connexion au réseau socionumérique restreinte au temps libre ou au temps privé de l'individu. Ce phénomène semble moins concerner les inactifs et les retraités. Par ailleurs, une frange, investie dans le monde associatif, retrouve souvent des temporalités d'activités quasiment équivalentes aux personnes présentes dans le monde du travail ou en cours d'études.

Bien qu'ils se servent de Facebook sur le lieu de travail ou d'études, les usagers sont contraints d'articuler leur usage en fonction de plusieurs temps dominants. Deux temps dominent les usages de Facebook parmi les personnes enquêtées: le temps scolaire et le temps professionnel. Le temps d'usage de Facebook se retrouve articulé entre deux oppositions: la première confronte le temps privé et le temps public, tandis que la seconde met en balance le temps de travail et le temps libre.

Dans le cas où des personnes sont amenées à faire usage de Facebook, particulièrement dans un cadre professionnel, certaines difficultés apparaissent. Cette volonté toujours plus forte de structurer et d'optimiser l'enchaînement des différentes temporalités de vie fait que la dissociation entre celles-ci devient de plus en plus laborieuse. La difficulté vient du fait que la temporalité d'usage de Facebook sillonne, en pointillé, les temporalités d'usage de l'informatique et d'Internet par des microconnexions régulières. Cette articulation se complexifie davantage lorsque les investissements personnel ou professionnel varient. Les technologies mobiles renforcent d'ailleurs ce phénomène en favorisant de multiples mais brèves connexions à Facebook. Cette pratique se définit souvent comme une dynamique permettant de combler le temps interstitiel, ce temps entre deux tâches, de non-activité. La temporalité d'usage de Facebook s'intègre dans un continuum de temporalités de vie et le parcourt, en pointillé, avec une logique de rationalisation du temps. De manière plus large, d'ailleurs, les TIC concourent de plus en plus fortement à la rationalisation des différentes temporalités de vie (Le Douarin, 2007).

Concernant l'incidence des rythmes de vie sur les pratiques de mise en visibilité de soi, l'accélération du temps, si ce n'est la perception du temps qui s'accélère, entraîne une indéniable révision des différents temps de vie. À côté de cela, suivant les périodes de vie, le rapport des individus au temps évolue, notamment lors de changement de situation entre l'activité professionnelle et l'inactivité. La modulation des rythmes de vie, amenant une révision des temporalités, induit une évolution des 
pratiques de mise en visibilité de soi. Le phénomène de rationalisation du temps conduit souvent à une superposition des temporalités.

Plus spécifiquement, la mise en visibilité numérique de soi semble s'apparenter à un jeu d'équilibre entre des pratiques de rationalisation de plus en plus importantes de cette exposition, donc basées sur l'usage de la raison dans une perspective utilitaire, et des pratiques fondées sur de l'intuitif et de l'émotionnel. Une approche dialectique de ces usages permet de comprendre cette oscillation du contrôle sur la visibilité de soi. D'un côté, les usages sont réfléchis, codifiés et anticipés et, de l'autre, les usages sont instables, fortement chargés émotionnellement et en perte de contrôle.

Dans cette recherche, les différentes temporalités de mise en visibilité se basent sur du déclaratif. Se faisant, la compréhension de ces temporalités dépend inévitablement de la conscience qu'ont les usagers du temps d'usage de Facebook. Nous pensons que le temps joue un rôle fondamental dans la construction et la modulation de ces pratiques et qu'il est donc possible d'élaborer une chronologie de mise en visibilité de soi sur Facebook. L'analyse des discours des personnes interrogées nous a permis de produire une chronologie de mise en visibilité de soi suivant quatre temporalités (Fig. 1). Ce modèle répond à une hypothèse selon laquelle les évolutions de la visibilité ne se réalisent pas de manière linéaire, mais plutôt qu'elles s'opèrent par phases et qu'elles sont concomitantes des diverses expériences d'usage.

\begin{tabular}{|c|c|c|c|}
\hline Temporalité 1 & Temporalité 2 & Temporalité 3 & Temporalité 4 \\
\hline $\begin{array}{c}\text { Acceptation de } \\
\text { l'invitation de mise } \\
\text { en visibilité de soi }\end{array}$ & $\begin{array}{c}\text { Résistance à l'invitation } \\
\text { et volonté de } \\
\text { préservation de l'intimité }\end{array}$ & $\begin{array}{c}\text { Tempérance vis-à- } \\
\text { vis de l'invitation }\end{array}$ & $\begin{array}{c}\text { Composition entre } \\
\text { opportunités et } \\
\text { risques }\end{array}$ \\
\hline \multicolumn{3}{|c|}{ ÉVOLUTION DES PRATIQUES DE MISE EN VISIBILITÉ DE SOI } \\
\hline $\begin{array}{c}\text { Forte mise en } \\
\text { visibilité de soi et } \\
\text { faible conscience } \\
\text { des engagements } \\
\text { liés à la visibilité }\end{array}$ & $\begin{array}{c}\text { Expérimentation de } \\
\text { l'exposition de soi et } \\
\text { conception de la }\end{array}$ & $\begin{array}{c}\text { Pisibilité informationnelle } \\
\text { visibilité et } \\
\text { acquisition de } \\
\text { compétence dans la } \\
\text { gestion de } \\
\text { confidentialité }\end{array}$ & $\begin{array}{c}\text { Régulation de la } \\
\text { visibilité et } \\
\text { coordination entre } \\
\text { exposition et } \\
\text { préservation }\end{array}$ \\
\hline
\end{tabular}

Figure 1. Chronologie de mise en visibilité de soi sur Facebook 
Une dialectique de la pudeur : les pratiques de mise en visibilité de soi sur Facebook

Sur ce continuum, la première temporalité correspond à l'émergence de formes d'exposition de soi avec l'acceptation du phénomène d'invitation à la mise en visibilité et à une faible conscience des engagements liés à cette pratique. La deuxième temporalité tient à des usages en construction avec une attitude de résistance par rapport à l'invitation, comprise comme la réalisation de la forte visibilité des informations personnelles, et à une volonté de préservation de l'intimité. La troisième temporalité renvoie à des usages en stabilisation avec une tempérance par rapport au phénomène d'invitation et à une acquisition de compétences dans la gestion des paramètres de confidentialité. La dernière temporalité se réfère à des usages coordonnés amenant à une gestion négociée des informations personnelles entre le visible et l'invisible. L'analyse des propos recueillis laisse apparaître que l'accumulation d'expériences sur Facebook conduit généralement à une plus grande aisance de la part des usagers avec le dispositif et, plus particulièrement, à une plus grande assurance dans l'administration de ce qu'ils exposent ou de ce qu'ils occultent.

II apparaît ainsi une pluralité de temporalités, articulées autour d'un ou plusieurs temps dominants, c'est-à-dire que ce temps dominant a nécessairement des incidences sur les différentes temporalités élaborées. Bien évidemment, cette chronologie correspond à un modèle tendanciel, elle est donc réductrice. Son but est de souligner le caractère dynamique des pratiques d'exposition de soi et de montrer qu'il s'opère une réorganisation, plus ou moins régulière, du visible par l'élaboration de stratégies suivant l'ancienneté d'usage. Ces évolutions dans la gestion des accès aux informations personnelles montrent qu'il s'opère des "glissements » de ces informations entre les différents espaces de visibilité. L'expérience d'usage, accumulée au cours du temps, est un facteur de variation de la taille des espaces de visibilité.

\section{Une évolution du cadre normatif de la pudeur}

II importe de rappeler que les pratiques d'exposition de soi sont préexistantes aux technologies numériques, tout comme celles dérogeant aux règles de la pudeur. L'impudeur est entendue comme un « risque pris par une personne quand elle ne réserve pas la monstration de certains de ses attributs identitaires à la sphère restreinte dans laquelle ils trouvent habituellement à s'exprimer » (Granjon et Denouël, 2010, p. 39). Ordinairement conçue comme une perte de contrôle,

tic\&société - 10(2-3), 2016-2017 
tout du moins détachée de la bienséance et animée par des pulsions, l'impudeur correspond à une pratique orientée vers la recherche de reconnaissance. Ce qui est appelé une prise de risque dans un contexte de mise en visibilité se rapporte à ce que Granjon (2011) nomme des «formes de reconnaissance de singularités subjectives ", c'est-à-dire des sollicitations de reconnaissances par autrui de ce qui fait le caractère particulier de l'usager. Pour autant, cette éventuelle augmentation de la réalisation de l'acte d'impudeur tient moins de la transgression que du jeu avec la limite en vue d'une reconnaissance positive par autrui (Roman, 1992).

Une analyse thématique des entretiens nous a permis de mettre au jour des mécanismes communs à un certain nombre de personnes que nous avons interrogées et, ainsi, de montrer que les facteurs d'invitation à l'exposition de soi correspondent, en grande partie, aux facteurs de préservation contre les potentielles atteintes à la vie privée. Les dispositifs qui invitent l'usager à se protéger sont aussi ceux qui le poussent à s'exposer. Ces facteurs sont au nombre de quatre. Le premier correspond à l'usager lui-même, par le biais des habitudes intériorisées. Selon le contexte d'interaction, la mise en visibilité se négociera en fonction des opportunités offertes et des risques encourus. Le deuxième facteur correspond au réseau de relations de l'usager imposant le respect des normes de sociabilité. Le réseau de relations concourt de la même manière à alimenter cette dynamique risque/opportunité. Le troisième facteur renvoie au dispositif sociotechnique Facebook intrinsèquement élaboré suivant cette attente de mise en visibilité de soi. Néanmoins, Facebook ne se positionne pas véritablement en agent de préservation de l'intimité, excepté lorsqu'une autorité judiciaire le contraint à adapter son système en fonction des règles de protection de la vie privée en vigueur dans le pays concerné. Finalement, le dernier facteur fait référence à la "société ", qui requiert que l'individu se conforme aux croyances, aux valeurs et aux normes sociales en vigueur.

À côté de cela, nos résultats nous ont permis de constater un double mécanisme de pénétration du privé par le public et de mise en visibilité du privé dans le public (Fig. 2). Le phénomène de pénétration du privé par le public doit être entendu comme une réduction des espaces intimes. Le public s'invite dans le privé. Les sollicitations permanentes à l'exposition de soi appellent à la réduction des espaces intimes. Cette intrusion du privé par le public a amené, dans certains cas, un durcissement des seuils de la pudeur et de la décence, tandis que le phénomène de mise en visibilité du privé dans le public 
Une dialectique de la pudeur : les pratiques de mise en visibilité de soi sur Facebook

correspond à une exposition variable de ce qui est lié à l'intimité et à l'intériorité de la personne. L'exemple des émissions de téléréalité permet, plus largement, de décrire ce phénomène de multiplication des actes perçus comme impudiques (Mehl, 2008).

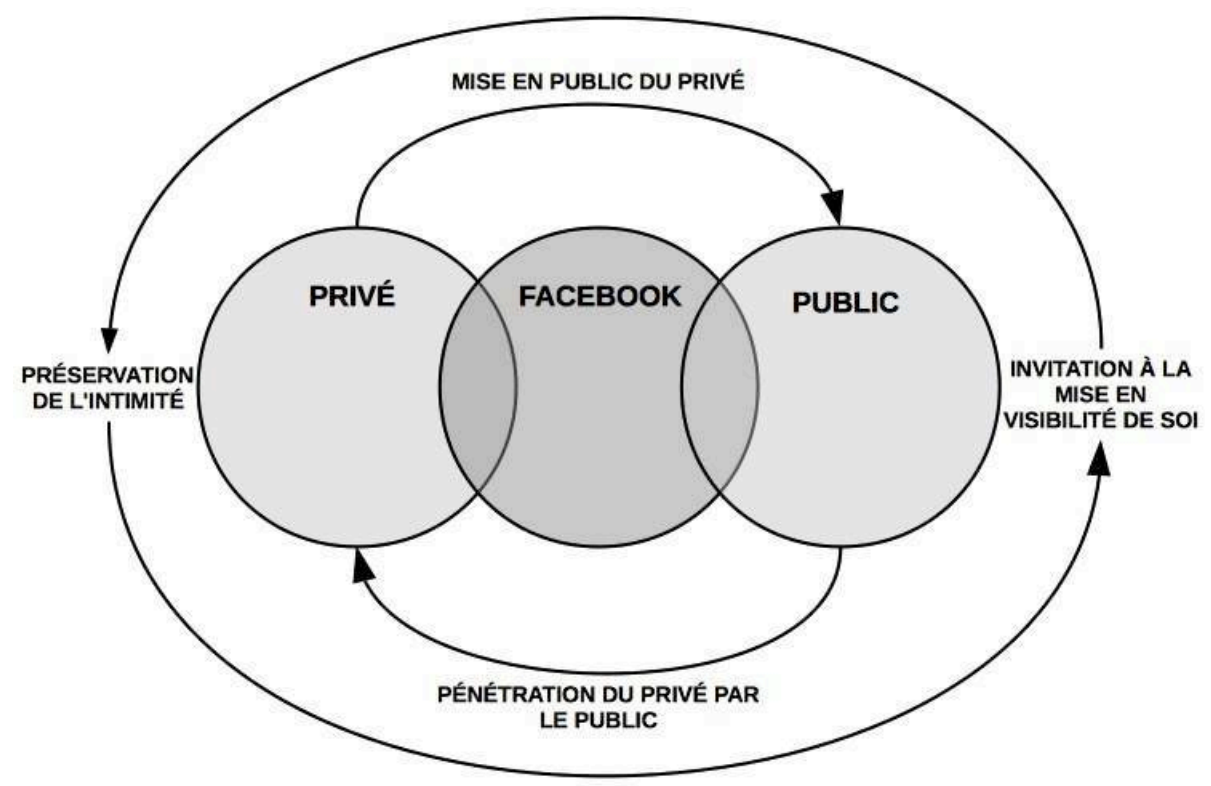

Figure 2. L'interpénétration du privé et du public

Un relâchement des individus face aux règles de la pudeur est possible dans le contexte d'une société où un minimum d'habitudes pudiques est assuré. L'évolution du rapport aux mœurs amène les individus à disposer d'une sensibilité d'autant plus importante à la pudeur, avec un renforcement de sa valeur et un seuil de tolérance aux actes impudiques tout aussi permissif. Les individus ne dévoilent pas davantage leur intimité qu'auparavant, mais l'intimité a acquis une plus forte valeur et la sensibilité à celle-ci s'en est accrue. La tolérance aux actes impudiques est plus grande du fait que les règles structurant les mœurs sont éminemment intériorisées.

L'hypothèse formulée par Isabelle Quéval (2012) à propos du rapport de l'homme avec son corps, et particulièrement concernant le souci de soi, peut être corrélée à cette question de l'évolution du rapport des individus aux normes de la pudeur. Elle avance l'idée que ce nouvel enjeu d'entretien du corps se situe au " point d'intersection entre une prescription collective - ne pas fumer, manger fruits et légumes, éviter le soleil, faire du sport, etc. - et une volonté personnelle - œuvrer 
au corps qui est soi -, [où] se noue la dimension originale d'une intériorisation » (Quéval, 2012, p. 31). Le phénomène de visibilité numérique se retrouve donc au cœur d'une dynamique, impliquant la pénétration du privé par le public et la mise en visibilité du privé dans le public, nourrie par une invitation collective au perfectionnement du corps et par un souci personnel de soi.

Toutefois, il reste tout de même difficile de définir le seuil de l'impudeur, ce moment où le dévoilement est excessif, puisqu'il est variable en fonction des acteurs et du contexte. Entre exaspération, incompréhension, condamnation ou acceptation, tolérance et sympathie, le rapport des personnes interrogées à l'égard des pratiques d'exposition de l'intimité est extrêmement variable. Effectivement, la mise en visibilité régulière de l'intime peut conduire des usagers à entretenir un rapport critique quant au dévoilement perçu comme excessif. La diversité des attitudes que ces usagers adoptent, tout du moins déclarent adopter, laisse poindre un phénomène plus général, à savoir que la tolérance à la mise en visibilité de l'intime accepte davantage d'excès, mais, passé le seuil de l'impudeur, la critique est d'autant plus acerbe.

Ce qui est régulièrement défini comme une perte de contrôle vis-à-vis des mœurs contemporaines est au cœur des dangers liés aux nouveaux modes d'interaction dans les espaces numériques, particulièrement sur les réseaux socionumériques. Prenant appui sur les propos de Cas Wouters (2003), Granjon (2014) use de la formule de contrôle du décontrôle des mœurs, comprenant qu'il y a un

« public, plus réflexif et distancié, dont la tolérance aux débordements du soi serait moins liée à un affermissement des autocontraintes qui pèse sur lui qu'à un apprentissage le rendant plus indulgent et compréhensif des singularités mises en scène au sein d'enceintes de moins en moins confinées et spécialisées » (Wouters, cité dans Granjon, 2014, p. 23-24).

La tolérance vis-à-vis de la transgression des règles structurant les mœurs semble plus importante, sans que les impudeurs excessives soient pour autant couramment acceptées. 
Une dialectique de la pudeur : les pratiques de mise en visibilité de soi sur Facebook

\section{Conclusion}

L'objectif de cette recherche vise à souligner le caractère contributif de ces pratiques à la dynamique relationnelle quotidienne. La mise en visibilité numérique de soi ne doit pas être disjointe des pratiques de sociabilité "hors-ligne " et renvoie à une diversification des modes de sociabilité, non à un repli sur soi dans l'espace numérique. Les réseaux socionumériques, entre autres dispositifs sociotechniques, ont entraîné une évolution de la vision normative des modes de sociabilité en coprésence.

La mise en visibilité de l'intime demeure peu généralisée sur Facebook. Il s'agit davantage de faire part de ses humeurs immédiates, tout en rejetant l'exposition d'une condition physique ou psychique dépréciée. Facebook se comprend notamment comme un espace d'immortalisation des moments importants du quotidien en excluant la narration de tous les instants de vie. La "condition physique dépréciée " renvoie, dans ce texte, à un corps dégradé, à un corps visiblement marqué par la vieillesse, la maladie, la saleté, le surpoids ou la détérioration physique. Sous certaines conditions, Facebook permet un plus grand contrôle sur son esthétique et s'apparente à un espace de négation, tout du moins un espace de refus du fétide, de la vieillesse, de la maladie, de la saleté, du gras et de l'infirmité. La mise en visibilité de caractéristiques spécifiques participerait d'une culture de l'esthétique, avec le jeune, le sain, le propre, le mince et le fort comme mécaniques de distinction. II serait intéressant d'interroger ce phénomène d'hygiène de la visibilité, entendu comme l'ensemble des mesures, des techniques et des procédés mis en œuvre pour préserver une "bonne " visibilité de soi. Plus encore, il importerait de mettre en lumière la morale régulant l'hygiène de la visibilité, c'est-à-dire expliquer l'ensemble des valeurs et des règles d'action préconisées pour ces pratiques de mise en visibilité de soi, mais aussi le rapport des usagers à cette morale (acceptation, transgression).

D'une manière générale, la mise en visibilité numérique des informations personnelles ne doit pas être comprise comme une pratique totalement dérégulée et incontrôlée. Elle ne déroge que marginalement au moralement acceptable. II apparaît bien un souci de régulation de la visibilité informationnelle sans pour autant que la pratique effective ne soit totalement maîtrisée. Non sans occulter les difficultés et les dangers rencontrés, les dérives et les drames observés, les individus disposent d'aptitudes quant à l'usage des dispositifs sociotechniques afin de réguler la visibilité numérique de soi. 
L'évolution du cadre normatif de la pudeur fait que les usages de ces dispositifs sociotechniques intègrent dorénavant davantage des jeux avec les règles régulant la mise en visibilité de soi, du corps et des sentiments.

Cette mise en discours de soi ne suit-elle pas une logique de type « Plus en parler pour mieux en parler » dans le sens moral du terme ? N'est-elle pas sujette à une mécanique de contrôle des énoncés et des énonciations, pour reprendre la formule de Foucault (1976), puisqu'en dernier recours, Facebook décide de l'acceptable et de l'intolérable ? Nous pouvons reprendre l'exemple de la régulière censure effectuée par Facebook sur le tableau de Gustave Courbet, L'origine du monde. Au final, ces pratiques de régulation de la visibilité informationnelle positionnent les réseaux socionumériques en de nouveaux espaces de sociabilité, mais dans quelle mesure ne contribuent-elles pas à renforcer ce phénomène d'hygiène de la visibilité de soi ?

\section{Références bibliographiques}

AGUITON C. et al., 2009, "Does showing off help to make friends? Experimenting a sociological game on self-exhibition and social networks ", ICWSM (International Conference on Weblogs and Social Media), 17-20 mai 2009, San Jose (États-Unis).

AKOUN A, 1998, Sociologie des communications de masse, Paris, Hachette.

ALLARD L. et VANDENBERGHE F., 2003, « Express yourself! Les pages perso. Entre légitimation technopolitique de l'individualisme expressif et authenticité réflexive peer to peer », Réseaux, n¹17, pp. 191-219.

ALTMAN I., 1977, « Privacy Regulation: Culturally Universal or Culturally Specific? », Journal of social issues, vol. 33, $\mathrm{n}^{\circ} 3$, pp. 66-84.

ARIĖS P. et DUBY G., 1999, Histoire de la vie privée. 1, De l'Empire romain à l'an mil, Paris, Le Seuil.

BEAUDE B., 2012, Internet: changer l'espace, changer la société, Paris, Éditions FYP. 
Une dialectique de la pudeur : les pratiques de mise en visibilité de soi sur Facebook

BOLOGNE J.-C., 1997, Histoire de la pudeur, Paris, Hachette.

BOYD D. et ELLISON N., 2007, "Social Network Sites: Definition, History, and Scholarship », Journal of ComputerMediated Communication, vol. 13, $\mathrm{n}^{\circ} 1$, pp. 210-230.

BOYD D. et HARGITTAI E., 2013, « Connected and Concerned: Variation in Parents' Online Safety Concerns », Policy \& Internet, vol. 5, $\mathrm{n}^{\circ} 3$, pp. 245-269.

BOYD D., 2014, It's Complicated: The Social Lives of Networked Teens, New Haven, Yale University Press.

BOYD D., 2008a, Taken Out of Context: American Teen Sociability in Networked Publics (thèse de doctorat), University of California, Berkeley, School of Information.

BOYD D., 2008b, « Facebook's Privacy Trainwreck: Exposure, Invasion, and Social Convergence ", Convergence, vol. 14, $\mathrm{n}^{\circ} 1$, pp. 13-20.

BOYD D., 2007, «Social Network Sites: Public, Private, or What?", Knowledge Tree, <http://www.danah.org/papers/KnowledgeTree.pdf>, dernière consultation le 16 mars 2017.

CARDON D. et DELAUNAY-TETEREL H., 2006, « La production de soi comme technique relationnelle. Un essai de typologie des blogs par leurs publics », Réseaux, n¹38, pp. 15-71.

CARDON D., 2008, "Le design de la visibilité. Un essai de cartographie du web 2.0 », Réseaux, n²152, pp. 93-137.

CHARTIER R., 1999, «Introduction », dans P. ARIÈS et G. DUBY (dir.), Histoire de la vie privée. 3. De la renaissance aux lumières, Paris, Le Seuil, pp. 161-165.

CORBIN A., COURTINE J.-J. et VIGARELLO G., 2005, Histoire du corps. 1. De la Renaissance aux lumières, Paris, Le Seuil.

COUTANT A. et STENGER T., 2010, « Processus identitaires et ordre de l'interaction sur les réseaux socionumériques ", Les enjeux de l'information et de la communication, $\mathrm{n}^{\circ} 1$, pp. $45-$ 64. 
DENOUËL J., 2011, «Identité », Communications, $\mathrm{n}^{\circ} 88$, pp. 75-82.

DUBAR C. et ROLLE C., 2008, "Les temporalités dans les sciences sociales: introduction ", Temporalités, $\mathrm{n}^{\circ} 8$, $<$ https://temporalites.revues.org/57>, dernière consultation le 16 mars 2017.

DUBAR C. et THOEMMES J., 2013, «Introduction. Les sciences sociales face aux temporalités », dans C. DUBAR et J. THOEMMES (dir.), Les temporalités dans les sciences sociales, Toulouse, Octarès, pp. 7-12.

DUBAR C., 2010, La crise des identités. L'interprétation d'une mutation, Paris, Presses universitaires de France.

ELIAS N., 1996, Du temps, Paris, Fayard.

ELIAS N., 1991, La société des individus, Paris, Fayard.

ELIAS N., 1973, La civilisation des mœurs, Paris, CalmannLévy.

FOUCAULT M., 1976, Histoire de la sexualité, Tome 1. La volonté de savoir, Paris, Gallimard.

FOUCAULT M., 1984, Histoire de la sexualité. Tome 3, Le souci de soi, Paris, Gallimard.

FRIED C., 1970, « Privacy », Yale Law Journal, vol. 77, pp. 475493.

FROOMKIN M., 2000, « The Death of Privacy? », Stanford Law Review, vol. 52, pp. 1461-1543.

GOFFMAN E., 1973, La mise en scène de la vie quotidienne. Tome 1, La présentation de soi, Paris, Les éditions de minuit.

GRANJON F. et DENOUËL J., 2010, «Exposition de soi et reconnaissance de singularités subjectives sur les sites de réseaux sociaux ", Sociologie, vol. 1, $n^{\circ} 1$, pp. 25-43.

GRANJON F., 2014, " Du (dé)contrôle de l'exposition de soi sur les sites de réseaux sociaux », Les cahiers du numérique, vol. $10, n^{\circ} 1$, pp. 19-44. 
Une dialectique de la pudeur : les pratiques de mise en visibilité de soi sur Facebook

GRANJON F., 2011, «De quelques pathologies sociales de l'individualité numérique. Exposition de soi et autoréification sur les sites de réseaux sociaux », Réseaux, $\mathrm{n}^{\circ} 167$, pp. 75103.

HEINICH N., 1997, La sociologie de Norbert Elias, Paris, La Découverte \& Syros.

ITO M. et al., 2008, Living and Learning with New Media: Summary of Findings from the Digital Youth Project, The John D. and Catherine T. MacArthur Foundation Reports on Digital Media and Learning, Chicago (États-Unis).

KANG J., 1998, « Information Privacy in Cyberspace Transactions », Stanford Law Review, vol. 50, pp. 1193-1294.

LE DOUARIN L., 2007, « “C'est personnel !”. L'usage des TIC par les cadres dans l'articulation des temps sociaux: vers une évolution de la rationalisation au travail ? ", L'Homme et la société, $\mathrm{n}^{\circ} 163-164$, pp. 75-94

LENHART A. et MADDEN M., 2007, « Teens, Privacy \& Online Social Networks: How Teens Manage Their Online Identities and Personal Information in the Age of MySpace ", Pew Internet and American Life Project, Washington (États-Únis).

LIVINGSTONE S., 2008, «Taking Risky Opportunities in Youthful Content Creation: Teenagers' Use of Social Networking Sites for Intimacy, Privacy and Self-Expression », New Media \& Society, vol. 10, n³, pp. 393-411.

MEHL D., 2008, "La télévision de l'intimité », Le temps des médias, $\mathrm{n}^{\circ} 10$, pp. 265-279.

PERROT M., 1999, « Introduction », dans P. ARIÈS et G. DUBY (dir.), Histoire de la vie privée. Tome 4, De la révolution à la Grande Guerre, Paris, Le Seuil, pp. 7-12.

POLIN R., 2000, « La culture proprement européenne au sein de la culture occidentale », dans P. TABATONI (dir.), La protection de la vie privée dans la société d'information. Tome 2, Paris, Presses universitaires de France, pp. 1-7.

QUEVAL I., 2012, «L'industrialisation de l'hédonisme. Nouveaux cultes du corps: de la production de soi à la perfectibilité addictive », Psychotropes, vol. 18, pp. 23-43. 
ROMAN J., 1992, " D'une impudeur, l'autre », dans C. HABIB (dir.), La pudeur. La réserve et le trouble, Paris, Autrement, pp. 66-71.

SAMUEL O., 2008, "Les démographes et le temps », Temporalités, $\quad \mathrm{n}^{\circ} 8, \quad<$ https://temporalites.revues.org/113>, dernière consultation le 16 mars 2017.

SENNETT R., 1979, Les tyrannies de l'intimité, Paris, le Seuil.

STONE E. et al., 1983, «A Field Experiment Comparing Information-Privacy Values, Beliefs, and Attitudes Across Several Types of Organizations ", Journal of Applied Psychology, vol. 68, $\mathrm{n}^{\circ}$ 3, pp. 459-468.

TABATONI P., 2002, « Indétermination et dynamique de la vie privée. Point de vue », dans P. TABATONI (dir.), La protection de la vie privée dans la société d'information. Tomes 3, 4 et 5, Paris, Presses universitaires de France, pp. 1-21.

TABATONI P., 2000, "Avant-propos », dans P. TABATONI (dir.), La protection de la vie privée dans la société d'information. Tome 1, Paris, Presses universitaires de France, pp. 3-7.

VINCENT G., 1999, "Postface », dans P. ARIÈS et G. DUBY (dir.), Histoire de la vie privée. 5. De la première guerre mondiale à nos jours, Paris, Le Seuil, pp. 549-590.

VOIROL O., 2005, « Visibilité et invisibilité : une introduction », Réseaux, $\mathrm{n}^{\circ}$ 129-130, pp. 9-36.

WARREN S. et BRANDEIS L., 1890, "The Right to Privacy », Harvard Law Review, vol. 4, n 5, pp. 193-220.

WESTIN A., 1970, Privacy and Freedom, New York, Atheneum.

WOUTERS C., 2003, "La civilisation des mœurs et des émotions: de la formalisation à l'informalisation », dans Y. BONNY, J.-M. DE QUEIROZ et E. NEVEU (dir.), Norbert Elias et la théorie de la civilisation. Lectures critiques, Rennes, Presses universitaires de Rennes, pp. 147-168. 